\title{
Implication of Season and Parity on Bioacoustics Features for Detection of Estrus Phase in Murrah Buffalo
}

\author{
Indu Devi ${ }^{1 *}$, Pawan Singh ${ }^{2}$, Kuldeep Dudi ${ }^{3}$ and Yajuvendra Singh ${ }^{4}$ \\ ${ }^{1}$ Livestock Production Management, ICAR-Central Institute for Research on Cattle, Meerut Cantt (UP), INDIA \\ ${ }^{2}$ Livestock Production Management Section, ICAR-National Dairy Research Institute, Haryana, INDIA \\ ${ }^{3}$ Animal Nutrition Division, ICAR-National Dairy Research Institute, Haryana, INDIA \\ ${ }^{4}$ Livestock Production Management, CVSc \& AH, DUVASU, Uttar Pradesh, INDIA
}

"Corresponding author: I Devi; E-mail: indulathwal@gmail.com

Received: 12 May, 2021

Revised: 28 June, 2021

Accepted: 10 July, 2021

\begin{abstract}
The present study was conducted to explore the season and parity effect on acoustic features of vocal signals articulated by Murrah buffaloes during estrous cycle. Voice and blood samples were collected from healthy Murrah buffaloes $(n=60)$, maintained at ICAR-NDRI, Karnal. Animals were classified into three groups heifers $(n=20)$, primiparous $(n=20)$, pluriparous $(n=20)$ and further each group (20) was classified into two sub groups $(n=10)$ based on winter (September-February) and summer (March-July) season. The acoustic features were extracted by the help of PRAAT 5.1.36 software package. The features like call duration ( $\mathrm{sec}$ ), intensity (mean, maximum, minimum) (dB), formants (Hz): F1, F2, F3, F4, F5, jitter (\%), shimmer (\%) and $\mathrm{N} / \mathrm{H}$ ratio $(\%)$ were found statistically $(P<0.05)$ different during estrus phase. The season effect especially during estrus phase was found significant $(P<0.05)$ for minimum intensity $(\mathrm{dB}), \mathrm{F} 1(\mathrm{~Hz}), \mathrm{F} 3(\mathrm{~Hz})$, and jitter $(\%)$. There was significant $(P<0.05)$ difference between heifer and pluriparous buffaloes for intensity $(\mathrm{dB})$ (mean, maximum, minimum), F1-F5 $(\mathrm{Hz})$ and jitter (\%). Significant differences were found for $\mathrm{E}_{2}(\mathrm{pg} / \mathrm{ml})$ and $\mathrm{P}_{4}(\mathrm{ng} / \mathrm{ml})$ plasma levels among four phases of cycle and between both seasons. This study concluded that hot season and parity cause variations in acoustic features like intensity, frequency and formants due to fluctuation in steroid hormones levels and summer stress. So, season and age factor might be considered for further determination of threshold values especially for estrus phase to develop algorithm/DSS for efficient and automatic estrus detection in buffaloes.
\end{abstract}

\section{HIGHLIGHTS}

(0 Need of animal friendly heat detection method for accurate identification of heat in buffalo.

( Vocal signatures-based acoustic features variation for estrus detection in buffalo.

Keywords: Acoustic feature, Buffalo, Estrus, Parity, Season

Buffalo is an important livestock species in Asia and other countries (Villanueva et al., 2018). The world buffalo population is approximately 199 million (FAOSTAT, 2012) and India is having milch buffalo population of 109.85 million (Livestock Census, 2019). To fulfil the increasing demand of dairy products, average herd size of dairy farms is continuously increasing across the world. In buffaloes, silent heat is main hurdle in attaining its full reproductive efficiency (Warriach et al., 2012) and missing of one estrus results in a loss of at least Rupees 373/animal/day (Kumar et al., 2013) and monthly amounts to a loss of about Rupees 4500-5000 to farmer (Srivastva et al., 2013). Although many methods (visual method, teaser bull, milk temperature, pedometer, activity meter, pressure sensing devices etc.) of heat detection

How to cite this article: Devi, I., Singh, P., Dudi, K. and Singh, Y. (2021). Implication of Season and Parity on Bioacoustics Features for Detection of Estrus Phase in Murrah Buffalo. J. Anim. Res., 11(04): 687-694.

Source of Support: None; Conflict of Interest: None क्) 
exist with different efficiency, but still there is need of animal friendly, cost effective, less laborious and efficient heat detection method for commercial buffalo farms. A recent area of interest with relatively little applied and explored use on a farm is vocalization or vocal behaviour which is non-invasive, objective and it is interesting to decode the information content of vocal signals for getting clues about estrus detection. The source-filter theory stated that vocal signals are produced by vibrations of vocal folds (source) which are eventually filtered by the vocal tract (filter). Vocalization can be linked to specific anatomical and physiological conditions of caller's vocal apparatus structures (Taylor and Reby, 2010). Previous studies (Chung et al., 2013; Lee et al., 2014; Rottgen et al., 2017) have proved that voice of female animals contain important cues about their reproductive status (fertile phase). Variation in vocal signals could be due to changes in hormonal status (Charlton et al., 2012) and it can give clue about vocaliser's size, age and reproductive status. Studies have proved that steroid hormones cause morphological changes in vocal folds and receptors for sex steroids have been found to be present on larynx layer (Voelter et al., 2008). Effect of developmental changes of body in different age groups on vocal patterns have been studied in goat (Briefer and McElligott, 2011) and calf (Jeon et al., 2009).

But dairy animals have received less attention about knowledge of the influence of factors such as environment, hormones, and age of animal on vocal parameters. In buffaloes, it is an established fact that during summer months decrease in expression of estrus signs and estrous cycle length occur less chances of successful conception, more silent heat cases (Prakash et al., 2005). So, hypothesis for this study was that as age and physiological state (estrus/heat) of animal change, its voice features also get changed due to sex steroid hormonal effect on voice production apparatus. Significant steroid hormonal level differences in summer and winter changes in buffaloes have been reported (Mondal et al., 2007). But there is dearth of studies regarding the effect of change in hormonal gradient on acoustic parameters in dairy buffaloes in different breeding seasons which is essentially needed to determine threshold levels of acoustic features to develop an algorithm system for automatic heat detection in buffaloes which can be useful throughout the year. Therefore, the present study was undertaken to explore the effect of parity and seasons on acoustic features during estrous cycle phases in buffaloes in order to identify estrus phase accurately.

\section{MATERIALS AND METHODS}

The study was carried out at Livestock Research Centre (LRC) of ICAR - National Dairy Research Institute, Karnal, Haryana, India. A subtropical climate normally prevails in Karnal. The maximum ambient temperature goes to $45{ }^{\circ} \mathrm{C}$ during summer and minimum about $2{ }^{\circ} \mathrm{C}$ during winter. The annual rainfall is about $760-960 \mathrm{~mm}$, most of which is received during June-July. Relative humidity of Karnal normally vary from 41-85 per cent but it remains high from July-September thus making hot-humid conditions for dairy buffaloes. The study was conducted from August 2017 to July 2018 therefore covering both winter (September-February) and summer (March-July) seasons effects on buffalo voice features.

\section{Animals grouping and management}

Healthy and reproductively sound Murrah buffaloes ( $n=60)$ were selected for voice recording. Animals were classified into three groups based on parity, including heifers $(n=20)$, primiparous buffaloes $(n=20)$ and pluriparous buffaloes $(n=20)$. Further animals $(20)$ of each group were classified into two sub groups, each of 10 animals, based on winter season and summer season. In all group's homogeneity was maintained in terms of parity and body weight. The mean \pm standard error of animal's age (months), body weight $(\mathrm{Kg})$ in first group: $29.17 \pm 0.71,450.30 \pm 9.32$; second group: $43.54 \pm 0.78,531.71 \pm 7.51$; third group: $73.59 \pm 1.79$, 608.26 \pm 8.98 , respectively. Animals under present study were housed in group under loose housing system and space requirements were provided as per BIS standards and feeding of animals was done as per NRC standards. All other management practices were followed without any interference.

\section{Collection of samples}

The voice and blood samples were collected from buffaloes only after confirming their estrus phase by visual and behavioural observation of estrus signs (Rajanarayanan and Archunan, 2011) and per-rectal examination for uterus tonicity for estrus phase by trained veterinarian. 
Buffaloes after 17 days of appearance in heat/estrus were continuously observed till next heat/estrus. As soon as buffalo was observed in estrus it was shifted to individual shed of dimension $6 \mathrm{~m} \times 3 \mathrm{~m}$. Their voice recording was performed in these sheds in order to avoid the chances of overlapping of their voice signals with other type of noise signals. Samples of voice were collected (covering four phases of estrous cycle) during morning hours between 6 to $8 \mathrm{AM}$ and evening hours between 5 to $7 \mathrm{PM}$ for a sufficient period of time so that at least 50 complete vocal signals could be recorded from each animal/day/ phase. Recording of sound signals from these buffaloes was executed using a video camera (Sony HDV FX7E, handicam) equipped with a good quality microphone (Sony ECM674, unidirectional). The microphone was fixed in the front wall of the recording shed at a height of $150 \mathrm{~cm}$ from floor, so that it could capture each and every activity of the buffalo under recording. Special care was taken so that no hindrance in natural behaviour of buffaloes could occur due to these recording devices. Moreover, due to unidirectional specification of microphone, very less other noise signals from the environment were recorded. After confirmation of second estrus, the collected samples were classified as proestrus ( -3 to -1 days), estrus ( 0 day), metestrus (1-3 days), and diestrus (10-17 days) phases (Perera, 2011). The recorded video data were saved in MTS File format while, audio data were saved in WAV file format. Blood sampling was done for estimation of estrogen $\left(\mathrm{E}_{2}\right)$ and progesterone hormones $\left(\mathrm{P}_{4}\right)$ in blood which in turn was used as a tool of confirmation/differentiation of four phases of estrous cycle through hormones. Blood samples $(5 \mathrm{ml})$ were collected on the defined days in heparinised vacutainer only after recording of voice samples. Thereafter, each blood sample was centrifuged at $4{ }^{\circ} \mathrm{C}$ at the rate of $3000 \mathrm{rpm}$ for 20 minutes to separate the plasma and changes in blood concentrations of estrogen $\left(\mathrm{E}_{2}\right)$ and progesterone hormones $\left(\mathrm{P}_{4}\right)$ were estimated with help of Bovine hormone ELISA kit.

\section{Editing, processing and extraction of voice features}

The recorded voice samples were edited by using Adobe Premium Pro-1.5 audio-visual software to remove superimposed voice signals and unnecessary noise signals. Voice signals were resampled at a sampling frequency of $48 \mathrm{KHz}$ and 16 bits to extract a given set of features. Extraction of acoustic features was done to generate the best set of parameters from all frames of each complete voice clip representing the voice signals. To convert waveform of voice signals to best parametric representation Mel-Frequency Cepstrum Coefficients (MFCCs) technique was used in present study (Davis and Mermelstein, 1980). The acoustic features (best set of parameters that represents the signals from all the frames of each voice clip) were extracted with the help of PRAAT 5.1.36 software package developed by Boersma and Weenink (2010). The acoustic features; Call Duration $(\mathrm{sec})$, Mean Intensity (dB), Maximum Intensity (dB), Minimum Intensity (dB), Formants (Hz) F1, F2, F3, F4, F5, Jitter (\%), Shimmer (\%), Noise/Harmonic ratio (\%), Number of pulses, Number of periods and Mean periods were extracted. Finally, numeric values of acoustic features extracted were arranged in MS Excel file format for the statistical analysis.

\section{Statistical analysis}

The data was analyzed by using least squares model by statistical package (SAS). The following least squares model was used in order to access the effect of estrous cycle phases, parity and seasons on vocal signals of particular individual animal.

$$
\begin{aligned}
Y_{i j k l}= & \mu+C_{i}+P_{j}+S_{k}+(C P)_{i j}+(C S)_{i k}+ \\
& (P S)_{j k}+(C P S)_{i j k}+e_{i j k l}
\end{aligned}
$$

Where, $Y_{i j k l}=$ Voice signal of $i^{\text {th }}$ phase of $j^{\text {th }}$ parity of $k^{\text {th }}$ season of $l^{\text {th }}$ animal, $\mu=$ Overall mean; $C_{i}=$ Effect of $i^{\text {th }}$ phase ( $i=$ proestrus, estrus, metestrus, diestrus); $P_{j}=$ Effect of $j^{\text {th }}$ parity ( $j=$ Heifer, primiparous, pluriparous); $S_{k}=$ Effect of $k^{\text {th }}$ season $(k=$ Winter and summer $) ;(C P)_{i j}=$ Interaction effect between $i^{\text {th }}$ phase and $j^{\text {th }}$ parity; $(C S)_{i k}=$ Interaction effect between $i^{\text {th }}$ phase and $k^{\text {th }}$ season; $(P S)_{j k}$ $=$ Interaction effect between $j^{\text {th }}$ parity and $k^{\text {th }}$ season; $(C P S)_{i j k}=$ Effect of interaction of $i^{\text {th }}$ phase, $j^{\text {th }}$ parity and $k^{\text {th }}$ season on $l^{\text {th }}$ animal; $e_{i j k l}=$ residual error with mean 0 and variance $\sigma^{2}$.

The significance of difference for least square means among various subclasses (pair-wise) was examined by using Duncan's Multiple Range Test (DMRT) as modified by Kramer (1957) with the use of inverse elements and root means squares for error. 


$$
\bar{Y}_{i}-\bar{Y}_{j} \times \sqrt{\frac{2}{C_{i i}+C_{j j}+C_{i j}}}
$$

If the values are greater than $\sigma^{2} e, Z(P, n e)$ then differences are said to be significant.

$\left(Y_{i}-Y_{j}\right)=$ Difference between two least squares means

$C_{i i}=$ Corresponding $i^{\text {th }}$ diagonal element of $C$ matrix

$C_{j j}=$ Corresponding $j^{\text {th }}$ diagonal element of $C$ matrix

$Z(P, n e)=$ Standardized range value in Duncan's table at the chosen level of probability for error degree of freedom

$P=$ Number of means involved in the comparison

$\sigma^{2} e=$ Root mean squares for error

\section{RESULTS AND DISCUSSION}

The least square mean values of $E_{2}$ hormone $(\mathrm{pg} / \mathrm{ml}), P_{4}$ hormone $(\mathrm{ng} / \mathrm{ml})$ hormone and different acoustic features during estrous cycle phases in summer and winter season has been presented in Table 1 and Table 2, respectively. There were significant differences for both hormones' levels among four phases of cycle in buffaloes and peak levels of $E_{2}(\mathrm{pg} / \mathrm{ml})(41.17 \pm 1.10 \mathrm{v} / \mathrm{s} 32.44 \pm 0.70)$ and $P_{4}$ $(\mathrm{ng} / \mathrm{ml}) \quad(3.90 \pm 0.14 \mathrm{v} / \mathrm{s} \quad 2.59 \pm 0.10)$ were significantly $(P<0.01)$ different between both seasons (Table 1). Out of total extracted acoustic features, call duration, intensity (mean, maximum, minimum), formants: $F 1, F 2, F 3, F 4$, $F 5$, jitter, shimmer and $\mathrm{N} / \mathrm{H}$ ratio were found statistically $(P<0.05)$ different/higher during estrus phase while no. of pulses $(P=0.902)$, no. of periods $(P=0.783)$ and mean periods $(P=0.405)$ were not found significantly different among four estrous cycle phase (Table 2). We could get inference that during estrus phase, duration of call increases, more energetic call (in form of intensity), more fundamental frequency, noisier sound and increase in asymmetric structures occur. While mean periods, no. of pulses and periods were not found significantly different among four phases of estrous cycle. Our finding corroborates with previous studies like Leong et al. (2003) in African elephant, Yeon et al. (2006); Schon et al. (2007), Chung et al. (2013); Dreschel et al. (2014); Lee et al. (2014) in cattle. The present study reported higher call duration and frequency of calls which might be due to positive effect of estrogen and antagonistic effect of progesterone (Pfefferele et al., 2011). Estrogen might be responsible for changes in acoustic features like frequency, intensity and noise to harmonics ratio. The structure of vocal calls seems to be strongly influenced and modulated by steroid (reproductive) hormones (Bryant and Haselton, 2009; Pfefferle et al., 2011) and this study is also based on the similar hypothesis. It has been found that the reproductive hormones cause morphological changes in vocal cords and larynx as their tissues has receptors for sex steroids (Newman et al., 2000; Schneider et al., 2007; Voelter et al., 2008) and thereby resulting changes in acoustic parameters of voice (Yeon et al., 2006).

But only four acoustic features (minimum intensity, F1, F3, jitter) showed significant difference especially during estrus phase between both seasons means seasonal fluctuations of steroid hormones affected only 'vocal tract' related voice features but not 'source' related voice parameters because short term transient changes in hormones in summer months may not be able to change vocal folds (source) morphology and tension for voice production. Estrogen and progesterone hormone were significantly reduced in during hot months (March-July) in comparison to mild/cold season (September-February) which increase chances of silent heat in buffaloes.

\section{Parity effect}

The least square means of acoustic features in different parities has been given in Table 3. If parity effect on acoustic features during estrus phase (because it is main important phase) is compared, we reported that there was significant $(P<0.05)$ difference between heifer and pluriparous buffaloes for intensity (dB) (mean, maximum, minimum), F1, F2, F3, F4, F5 (Hz), jitter (\%) (Table 3). Although call duration ( $\mathrm{sec}$ ) during estrus/heat period was found longer in pluriparous (fully mature) buffaloes in comparison to primiparous and heifers although not statistically significant. Because call duration is related to the age, the lung capacity is closely related to body size of animals (Fitch and Hauser, 2002; Feinberg et al., 2005) which is more in fully mature adult pluriparous buffaloes. Intensity $(\mathrm{dB})$ represents energy in vocalization or logarithm of amplitude. Pluriparous buffaloes had significantly more intensity (mean, maximum, minimum) in comparison to heifers because adult animal produce sound with more aggression and energy due to more estrogen level and experience. Formants of voice represent 
Table 1: Least square means \pm S.E of hormones in summer and winter seasons during four phases of estrous cycle

\begin{tabular}{llllll}
\hline \multirow{2}{*}{ Hormone } & \multirow{2}{*}{ Seasons } & \multicolumn{4}{c}{ Phases of estrous cycle } \\
\cline { 3 - 6 } & Proestrus & Estrus & Metestrus & Diestrus \\
\hline Estrogen $(\mathrm{pg} / \mathrm{ml})$ & Summer & $32.44^{\mathrm{aq}} \pm 0.70$ & $16.91^{\mathrm{bq}} \pm 0.56$ & $4.84^{\mathrm{d}} \pm 0.45$ & $7.86^{\mathrm{c}} \pm 0.61$ \\
& Winter & $41.17^{\mathrm{ap}} \pm 1.10$ & $22.42^{\mathrm{bp}} \pm 0.68$ & $5.55^{\mathrm{d}} \pm 0.38$ & $7.05^{\mathrm{c}} \pm 0.58$ \\
& Overall mean & $36.80^{\mathrm{a}} \pm 1.03$ & $20.67^{\mathrm{b}} \pm 0.54$ & $5.20^{\mathrm{d}} \pm 0.30$ & $7.46^{\mathrm{c}} \pm 0.42$ \\
\hline Progesterone $(\mathrm{ng} / \mathrm{ml})$ & Summer & $0.82^{\mathrm{b}} \pm 0.05$ & $0.36^{\mathrm{d}} \pm 0.02$ & $0.64^{\mathrm{c}} \pm 0.04^{\mathrm{c}}$ & $2.59^{\mathrm{aq}} \pm 0.10$ \\
& Winter & $1.01^{\mathrm{b}} \pm 0.09$ & $0.42^{\mathrm{d}} \pm 0.03$ & $0.63^{\mathrm{c}} \pm 0.03^{\mathrm{c}}$ & $3.90^{\mathrm{ap}} \pm 0.14$ \\
& Overall mean & $0.92^{\mathrm{b}} \pm 0.06$ & $0.39^{\mathrm{d}} \pm 0.02$ & $0.64^{\mathrm{c}} \pm 0.02$ & $2.99^{\mathrm{a}} \pm 0.11$ \\
\hline
\end{tabular}

Means bearing different superscripts $(a, b, c, d)$ differ significantly across rows $(P<0.01)$, means bearing different superscripts $(p, q)$ differ significantly across columns $(P<0.01)$.

Table 2: Least square means \pm S.E of acoustic features in different seasons during four phases of estrous cycle

\begin{tabular}{|c|c|c|c|c|c|}
\hline \multirow{2}{*}{ Acoustic features } & \multirow{2}{*}{ Seasons } & \multicolumn{4}{|c|}{ Phases of estrous cycle } \\
\hline & & Proestrus & Estrus & Metestrus & Diestrus \\
\hline \multirow[t]{2}{*}{ Call duration (sec) } & Summer & $0.96^{\mathrm{c}} \pm 0.04$ & $2.84^{\mathrm{a}} \pm 0.09$ & $0.81^{\mathrm{c}} \pm 0.04$ & $1.41^{\mathrm{b}} \pm 0.60$ \\
\hline & Winter & $1.12^{\mathrm{b}} \pm 0.12$ & $2.94^{\mathrm{a}} \pm 0.08$ & $0.87^{b} \pm 0.04$ & $0.83^{b} \pm 0.04$ \\
\hline \multirow{2}{*}{$\begin{array}{l}\text { Mean Intensity } \\
(\mathrm{dB})\end{array}$} & Summer & $67.06^{\mathrm{bq}} \pm 0.58$ & $78.93^{a} \pm 0.77$ & $67.07^{b} \pm 0.61$ & $66.16^{\mathrm{b}} \pm 0.56$ \\
\hline & Winter & $70.70^{\mathrm{bp}} \pm 0.99$ & $79.71^{\mathrm{a}} \pm 0.55$ & $66.76^{\mathrm{c}} \pm 0.60$ & $67.32^{c} \pm 0.68$ \\
\hline \multirow{2}{*}{$\begin{array}{l}\text { Maximum } \\
\text { intensity (dB) }\end{array}$} & Summer & $71.47^{\mathrm{b}} \pm 1.96$ & $114.48^{\mathrm{a}} \pm 26.15$ & $71.76^{\mathrm{b}} \pm 0.58$ & $72.47^{b} \pm 0.62$ \\
\hline & Winter & $75.01^{\mathrm{b}} \pm 0.78$ & $86.52^{b} \pm 0.70$ & $74.96^{\mathrm{b}} \pm 0.73$ & $75.98^{\mathrm{a}} \pm 1.96$ \\
\hline \multirow{2}{*}{$\begin{array}{l}\text { Minimum } \\
\text { intensity (dB) }\end{array}$} & Summer & $57.85^{\text {bq }} \pm 0.68$ & $63.67^{\mathrm{ap}} \pm 0.93$ & $58.17^{\mathrm{bq}} \pm 0.56$ & $57.83^{b} \pm 0.56$ \\
\hline & Winter & $62.74^{\text {ap }} \pm 0.69$ & $61.38^{\mathrm{aq}} \pm 0.99$ & $60.25^{\mathrm{bp}} \pm 0.85$ & $60.39^{\mathrm{b}} \pm 0.65$ \\
\hline \multirow[t]{2}{*}{ Formant $1(\mathrm{~Hz})$} & Summer & $1001.09^{\mathrm{bq}} \pm 16.42$ & $1413.60^{\mathrm{aq}} \pm 16.76$ & $962.24^{\mathrm{dq}} \pm 10.49$ & $1000.54^{\mathrm{b}} \pm 18.72$ \\
\hline & Winter & $1055.14^{\mathrm{bp}} \pm 30.13$ & $1471.89^{\mathrm{ap}} \pm 22.45$ & $997.70^{\mathrm{cp}} \pm 11.25$ & $1007.85^{\mathrm{c}} \pm 16.42$ \\
\hline \multirow[t]{2}{*}{ Formant $2(\mathrm{~Hz})$} & Summer & $2060.97^{b} \pm 32.90$ & $2732.22^{\mathrm{a}} \pm 21.98$ & $2028.06^{\mathrm{b}} \pm 28.25$ & $2053.75^{\mathrm{b}} \pm 21.62$ \\
\hline & Winter & $2128.54^{b} \pm 53.99$ & $2719.21^{\mathrm{a}} \pm 35.72$ & $1995.33^{d} \pm 29.68$ & $2010.31 \mathrm{c} \pm 32.90$ \\
\hline \multirow[t]{2}{*}{ Formant $3(\mathrm{~Hz})$} & Summer & $3646.71^{\mathrm{bp}} \pm 20.67$ & $3934.59^{\mathrm{ap}} \pm 53.29$ & $3624.45^{\mathrm{bp}} \pm 35.66$ & $3698.92^{\mathrm{bp}} \pm 43.45$ \\
\hline & Winter & $3537.72^{\mathrm{bq}} \pm 56.63$ & $3897.24^{\mathrm{aq}} \pm 62.07$ & $3451.93^{\mathrm{bq}} \pm 72.25$ & $3491.25^{\mathrm{bq}} \pm 48.08$ \\
\hline \multirow[t]{2}{*}{ Formant $4(\mathrm{~Hz})$} & Summer & $4950.82^{b} \pm 110.39$ & $5630.83^{\mathrm{a}} \pm 141.49$ & $5008.12^{b} \pm 20.09$ & $4951.90^{\mathrm{b}} \pm 29.93$ \\
\hline & Winter & $4985.61^{b} \pm 49.37$ & $5728.20^{\mathrm{a}} \pm 51.58$ & $4944.99^{c} \pm 69.43$ & $5000.42^{b} \pm 110.39$ \\
\hline \multirow[t]{2}{*}{ Formant $5(\mathrm{~Hz})$} & Summer & $6337.19^{\mathrm{b}} \pm 42.46$ & $6632.42^{\mathrm{a}} \pm 161.78$ & $5981.76^{\mathrm{b}} \pm 55.71$ & $6038.72^{b} \pm 29.49$ \\
\hline & Winter & $6208.65^{\mathrm{b}} \pm 74.09$ & $6923.22^{\mathrm{a}} \pm 39.67$ & $6280.31^{b} \pm 391.89$ & $5911.13^{b} \pm 42.46$ \\
\hline \multirow[t]{2}{*}{ Jitter (\%) } & Summer & $2.43^{\mathrm{bp}} \pm 0.38$ & $7.45^{\mathrm{aq}} \pm 0.47$ & $3.12^{\mathrm{bq}} \pm 0.32$ & $3.07^{\mathrm{bq}} \pm 0.38$ \\
\hline & Winter & $3.01^{\mathrm{bp}} \pm 0.46$ & $11.09^{\mathrm{ap}} \pm 1.24$ & $4.12^{b p} \pm 0.69$ & $3.89^{\mathrm{bp}} \pm 0.69$ \\
\hline \multirow[t]{2}{*}{ Shimmer (\%) } & Summer & $9.34^{b} \pm 0.40$ & $22.46^{\mathrm{a}} \pm 0.69$ & $9.14^{b} \pm 0.51$ & $10.06^{\mathrm{b}} \pm 0.48$ \\
\hline & Winter & $11.97^{b} \pm 0.86$ & $21.44^{\mathrm{a}} \pm 1.21$ & $11.96^{\mathrm{b}} \pm 0.87$ & $13.42^{b} \pm 1.30$ \\
\hline \multirow{2}{*}{$\begin{array}{l}\text { Noise/Harmonic } \\
\text { ratio (\%) }\end{array}$} & Summer & $4.56^{\mathrm{b}} \pm 0.49$ & $13.12^{\mathrm{a}} \pm 0.87$ & $3.76^{b} \pm 0.31$ & $4.38^{b} \pm 0.30$ \\
\hline & Winter & $5.12^{\mathrm{b}} \pm 0.46$ & $14.63^{\mathrm{a}} \pm 1.18$ & $3.68^{b} \pm 0.38$ & $4.07^{b} \pm 0.56$ \\
\hline \multirow[t]{2}{*}{ Number of pulses } & Summer & $97.50 \pm 12.26$ & $102.45 \pm 23.60$ & $79.40 \pm 11.46$ & $96.07 \pm 10.33$ \\
\hline & Winter & $106.14 \pm 18.17$ & $113.42 \pm 32.55$ & $103.54 \pm 13.32$ & $86.58 \pm 16.10$ \\
\hline \multirow{4}{*}{$\begin{array}{l}\text { Number of } \\
\text { periods } \\
\text { Mean periods }\end{array}$} & Summer & $93.42 \pm 12.26$ & $96.40 \pm 22.75$ & $96.40 \pm 11.17$ & $91.61 \pm 10.23$ \\
\hline & Winter & $102.15 \pm 17.67$ & $108.56 \pm 31.38$ & $98.45 \pm 13.16$ & $82.41 \pm 15.81$ \\
\hline & Summer & $0.009 \pm 0.00$ & $0.010 \pm 0.01$ & $0.009 \pm 0.01$ & $0.009 \pm 0.01$ \\
\hline & Winter & $0.010 \pm 0.00$ & $0.008 \pm 0.01$ & $0.008 \pm 0.01$ & $0.008 \pm 0.01$ \\
\hline
\end{tabular}

Values bearing different superscripts $(\mathrm{a}, \mathrm{b}, \mathrm{c})$ differ significantly across rows $(P<0.05)$, Values bearing different superscripts (p,q) differ significantly across columns $(P<0.05)$. 
Table 3: Least square Means \pm S.E of acoustic features in different parities during estrus phase

\begin{tabular}{|c|c|c|c|c|}
\hline \multirow{2}{*}{ Acoustic features } & \multirow{2}{*}{ Seasons } & \multicolumn{3}{|c|}{ Parity of buffalo } \\
\hline & & Heifer & Primiparous & Pluriparous \\
\hline \multirow{2}{*}{ Call duration (sec) } & Summer & $0.57 \pm 0.19$ & $1.12 \pm 0.47$ & $1.58 \pm 0.20$ \\
\hline & Winter & $0.99 \pm 0.18$ & $1.52 \pm 0.47$ & $1.88 \pm 0.20$ \\
\hline \multirow{2}{*}{ Mean intensity (dB) } & Summer & $63.45^{\mathrm{bq}} \pm 0.75$ & $69.81^{\mathrm{aq}} \pm 0.68$ & $77.85^{\mathrm{aq}} \pm 0.47$ \\
\hline & Winter & $70.19^{\mathrm{bp}} \pm 0.88$ & $79.11^{\mathrm{ap}} \pm 1.41$ & $84.49^{\mathrm{ap}} \pm 0.16$ \\
\hline \multirow{2}{*}{ Maximum intensity (dB) } & Summer & $63.45^{\mathrm{bq}} \pm 0.68$ & $77.85^{\mathrm{aq}} \pm 0.47$ & $79.85^{\mathrm{aq}} \pm 0.20$ \\
\hline & Winter & $70.19^{\mathrm{bp}} \pm 0.76$ & $71.12^{\mathrm{bp}} \pm 0.88$ & $79.11^{\mathrm{ap}} \pm 1.41$ \\
\hline \multirow{2}{*}{ Minimum intensity (dB) } & Summer & $51.35^{\mathrm{b}} \pm 0.92$ & $56.89^{\mathrm{a}} \pm 0.80$ & $59.42^{\mathrm{a}} \pm 0.67$ \\
\hline & Winter & $50.29^{b} \pm 0.70$ & $58.07^{\mathrm{a}} \pm 0.86$ & $58.90^{\mathrm{a}} \pm 0.58$ \\
\hline \multirow{2}{*}{ Formant $1(\mathrm{~Hz})$} & Summer & $1355.98^{\mathrm{aq}} \pm 51.89$ & $1100.67^{\mathrm{abq}} \pm 37.39$ & $882.59^{\mathrm{bp}} \pm 42.50$ \\
\hline & Winter & $1555.56^{\text {ap }} \pm 41.14$ & $1312.51^{\mathrm{bp}} \pm 55.13$ & $1087.59^{\mathrm{bp}} \pm 46.39$ \\
\hline \multirow{2}{*}{ Formant $2(\mathrm{~Hz})$} & Summer & $2602.32^{\mathrm{a}} \pm 72.53$ & $2240.01^{\mathrm{ab}} \pm 66.16$ & $1915.17^{b} \pm 74.73$ \\
\hline & Winter & $2836.36^{\mathrm{a}} \pm 62.85$ & $2102.09^{\mathrm{ab}} \pm 93.98$ & $2213.75^{b} \pm 66.96$ \\
\hline \multirow{2}{*}{ Formant $3(\mathrm{~Hz})$} & Summer & $3647.70^{\text {ap }} \pm 27.69$ & $3089.04^{b} \pm 42.89$ & $3059.17^{\mathrm{bp}} \pm 53.45$ \\
\hline & Winter & $3942.95^{\mathrm{aq}} \pm 27.69$ & $3671.64^{\mathrm{bq}} \pm 42.89$ & $3169.59^{\mathrm{bq}} \pm 74.97$ \\
\hline \multirow{2}{*}{ Formant $4(\mathrm{~Hz})$} & Summer & $5512.71^{\mathrm{a}} \pm 97.61$ & $5128.61^{\mathrm{b}} \pm 93.31$ & $4764.94^{b} \pm 77.88$ \\
\hline & Winter & $5763.81^{\mathrm{a}} \pm 94.22$ & $5435.81^{\mathrm{b}} \pm 102.19$ & $4952.70^{b} \pm 96.18$ \\
\hline \multirow{2}{*}{ Formant $5(\mathrm{~Hz})$} & Summer & $6824.87^{\mathrm{a}} \pm 92.73$ & $6070.45^{\mathrm{b}} \pm 114.49$ & $6247.26^{\mathrm{b}} \pm 287.88$ \\
\hline & Winter & $7175.03^{a} \pm 290.6$ & $6439.19^{b} \pm 117.30$ & $5764.54^{b} \pm 96.12$ \\
\hline \multirow{2}{*}{ Jitter (\%) } & Summer & $5.27^{\mathrm{ap}} \pm 0.12$ & $3.95^{\mathrm{aq}} \pm 0.42$ & $3.83^{\mathrm{bq}} \pm 0.52$ \\
\hline & Winter & $6.66^{\mathrm{aq}} \pm 0.52$ & $6.60^{\mathrm{ap}} \pm 1.06$ & $3.33^{\mathrm{bp}} \pm 1.12$ \\
\hline \multirow{2}{*}{ Shimmer (\%) } & Summer & $13.05^{\mathrm{q}} \pm 1.37$ & $13.09^{\mathrm{q}} \pm 1.19$ & $12.12^{\mathrm{q}} \pm 1.52$ \\
\hline & Winter & $14.32^{\mathrm{p}} \pm 1.28$ & $15.32^{\mathrm{p}} \pm 1.40$ & $13.44^{\mathrm{p}} \pm 1.09$ \\
\hline \multirow{2}{*}{ Noise/Harmonic ratio (\%) } & Summer & $6.00 \pm 0.70$ & $6.33 \pm 1.06$ & $7.04 \pm 1.16$ \\
\hline & Winter & $7.10 \pm 1.05$ & $6.98 \pm 1.30$ & $6.53 \pm 1.24$ \\
\hline \multirow{2}{*}{ No of pulses } & Summer & $104.38 \pm 11.43$ & $83.7 \pm 13.95$ & $93.92 \pm 14.05$ \\
\hline & Winter & $101.41 \pm 15.42$ & $124.78 \pm 24.34$ & $81.06 \pm 11.97$ \\
\hline \multirow{2}{*}{ No of periods } & Summer & $98.81 \pm 10.89$ & $78.76 \pm 13.78$ & $90.26 \pm 13.65$ \\
\hline & Winter & $97.83 \pm 14.93$ & $119.01 \pm 23.69$ & $76.85 \pm 11.58$ \\
\hline \multirow{2}{*}{ Mean periods } & Summer & $0.009 \pm 0.0006$ & $0.009 \pm 0.0005$ & $0.009 \pm 0.0005$ \\
\hline & Winter & $0.010 \pm 0.0006$ & $0.008 \pm 0.0004$ & $0.008 \pm 0.0004$ \\
\hline
\end{tabular}

Values bearing different superscripts $(\mathrm{a}, \mathrm{b}, \mathrm{c})$ differ significantly across rows $(P<0.05)$, Values bearing different superscripts $(\mathrm{p}, \mathrm{q})$ differ significantly across columns $(P<0.05)$.

resonant frequencies at which the power of fundamental frequency reaches the local maximum value (Fitch, 2000). Values for the first five lowest resonant frequency or formants $(F 1, F 2, F 3, F 4, F 5)$ were examined in this study. Formants were found significantly higher in heifer group as compared to primiparous and pluriparous buffaloes. The effective length of the vocal tract determines the overall spacing of formant frequencies and longer, thicker vocal tracts of adult animals produce lower formants (Fitch and Hauser, 2002). So, these acoustic parameters can provide reliable information about caller age, specifically fundamental frequency. Jitter represents cycle to cycle frequency variation of voice and was found more in adult pluriparous buffaloes because adult buffaloes had more experience in modulating their voice frequency. The higher levels of steroid hormones in adult animals may be responsible for these differences in some voice features (Evans et al., 2008; Bruckert, 2010) because vocal fold mass, stiffness and length get changed. It clearly indicates that voice signals of animals definitely change as per changes in age which might be a response to anatomical and hormonal change taking place in animals during growing stage. If parity wise season effect is considered, we reported that intensity (mean, maximum, minimum), 
$F 1, F 3$, jitter, shimmer; were statistically different between both seasons but irrespective of parity. Thereby, parity wise seasonal effect is not significant but age of animal is more important factor to define standard threshold values for estrus phase detection.

\section{CONCLUSION}

This study reported that season has some effect on intensity, frequency and formants due to fluctuation in steroid hormones levels and heat stress induced discomfort during summer season. Age of animals also affect peculiarity of voice parameters. So, in order to determine threshold levels of important acoustic features for estrus detection, season and age of animal should be taken into consideration for practical application of acoustic sensing for estrus detection in buffalo farms. However, further studies are required to further explore the seasonal variations in acoustic features of animals by considering the different management practices during summer season to be followed in field conditions because season has important part to play in buffalo reproduction under tropical environment.

\section{ACKNOWLEDGMENTS}

The authors sincerely thank Director, ICAR-National Dairy Research Institute, Karnal for providing financial help and facilities to carry out this work successfully.

\section{REFERENCES}

Boersma, P. and Weenink, D. 2010. PRAAT: Doing Phonetics by Computer. Phonetic Sciences. University of Amsterdam, Spuistraat, 210, Netherland.

Briefer, E. and McElligott, A. . 2011. Indicators of age, body size and sex in goat kid calls revealed using the source-filter theory. Appl. Anim. Behav. Sci., 133: 175-185.

Bruckert, L., Bestelmeyer, P., Latinus, M., Rouger, J., Charest, I., Rousselet, G.A., Kawahara, H. and Belin, P. 2010. Vocal Attractiveness Increases by Averaging. Curr. Biol., 20: 116120.

Bryant, G. and Haselton, M. 2009. Vocal cues of ovulation in human females. Biol. Lett., 5: 12-15.

Charlton, B.D., Swaisgood, R.R., Zhihe, Z. and Snyder, R.J. 2012. Giant pandas attend to androgen-related variation in male bleats. Behav. Ecol. Sociobiol., 66: 969-974.
Chung, Y., Lee. J., Park, D., Chang, H.H. and Kim, S. 2013. Automatic detection of cow's oestrus in audio surveillance system. Asian Australas. J. Anim. Sci., 26: 1030-1037.

Davis, S. and Mermelstein, P. 1980. Comparison of parametric representations for monosyllabic word recognition in continuously spoken sentences. In IEEE transactions on acoustics, speech, and signal processing, 28: 357-366.

Dreschel, S., Schön, P.C., Kanitz, W. and Mohr, E. 2014. Vocalization of dairy cattle during the oestrous cycle in two different housing systems. Züchtungskunde., 86: 157-169.

Evans, S., Neave, N., Wakelin, D. and Hamilton, C. 2008. The relationship between testosterone and vocal frequencies in human males, Physiol. Behav., 93: 783-788.

FAOSTAT., 2012. FAOSTAT Agricultural Data. Food and Agriculture Organization Statistics, Rome, Italy.

Feinberg, D.R., Jones, B.C., Little, A.C., Burt, D.M. and Perrett, D.I. 2005. Manipulations of fundamental and formant frequencies influence the attractiveness of human male voices. Anim. Behav., 69: 561-568.

Fitch, W.T. 2000. The phonetic potential of nonhuman vocal tracts: Comparative cineradiographic observations of vocalizing animals. Phonetica., 57: 205-218.

Fitch, W.T. and Hauser, M.D. 2002. Unpacking 'honesty:' vertebrate vocal production and the evolution of acoustic signals in Acoustic Communication, edited by A. Simmons, Fay, R. ., Popper, A.N., Springer-Verlag, New York. 65-137.

Jeon, J.H., Song, J.I. and Kim, D.H. 2009. A note on acoustic analysis of dairy calves' vocalizations at 1 day after separation from dam. Ital. J. Anim. Sci., 8: 113-119.

Jones, G. and Ransome, R. 1993. Echolocation Calls of Bats are Influenced by Maternal Effects and Change over a Lifetime. Proc. R. Soc. Lond. Biol., 252: 125-128.

Kramer, C.Y. 1957. Extension of multiple range tests to group correlated adjusted means. Biometrics., 13: 13-18.

Kumar, P.R., Shukla, S.N., Shrivastava, O.P. and Purkayastha, R.D., 2013. Incidence of postpartum anestrus among buffaloes in and around Jabalpur. Vet. World, 6: 716-9.

Lee, J., Zuo, S., Chung, Y. and Park, D. 2014. Formant-based Acoustic Features for Cow's Estrus Detection in Audio Surveillance System. $11^{\text {th }}$ IEEE International Conference on Advanced Video and Signal Based Surveillance (AVSS).

Leong, K.M., Ortolani, A., Graham, L.H. and Savage, A. 2003. The use of low frequency vocalizations in African elephant (Loxodonta africana) reproductive strategies. Horm. Behav., 43: 433-443.

Livestock Census $\left(20^{\text {th }}\right) .2019$. Animal Husbandry Statistics. Department of Animal Husbandry and Dairying, Ministry of Fisheries, Animal Husbandry \& Dairying. Government of India, Krishi Bhawan, New Delhi, India. 
Madan, M.L. 1990. Factors limiting superovulation responses in embryo transfer programme among buffaloes. Theriogenology, 33: 280.

Mondal, S., Prakash, B.S. and Palta, P. 2007. Endocrine Aspects of Oestrous Cycle in Buffaloes (Bubalus bubalis): An Overview. Asian-Aust. J. Anim. Sci., 20: 124-131.

Perera, B.M.A.O. 2011. Reproductive cycles of buffalo. Anim. Reprod. Sci., 124: 194-199.

Pfefferle, D., Heistermann, M., Pirow, R., Hodges, J.K. and Fischer, J. 2011. Estrogen and progestogen correlates of the structure of female copulation calls in semi-free-Ranging barbary macaques (Macaca sylvanus). Int. J. Primatol., 32: 992-1006.

Prakash, B.S., Sarkar, M., Paul, V., Mishra, D.P., Mishra, A. and Meyer, H.H.D. 2005. Postpartum endocrinology and prospects for fertility improvement in the lactating riverine buffalo (Bubalus bubalis) and yak (Poephagus grunniens L.). Livest. Sci., 98:13-23

Quinlan, J.R. 1993. C4.5: Programs for Machine Learning, Morgan Kaufmann, Los Altos.

Rajanarayanan S. and Archunan, G. 2011. Identification of urinary sex pheromones in female buffaloes and their influence on bull reproductive behaviour. Res. Vet. Sci., 91: 301-305.

Reby, D., Charlton, D.B., Locatelli, Y. and McComb, K. 2010. Oestrus red deer hinds prefers male roars with higher fundamental frequencies. Proc. R. Soc. London Biol., 277: 2747-2753.

Rottgen, V., Becker, F., Tuchscherer, A., Wrenzycki, C., Düpjan, S., Schön, P.C. and Puppe, B., 2018. Vocalization as an indicator of estrus climax in Holstein heifers during natural estrus and superovulation. J. Dairy Sci., 101: 1-12.
Roy, D.J., Bhattacharya, A.R. and Luktuke, S.N. 1972., Oestrus and ovarian activities of buffaloes in different months. Indian Vet. J., 49: 54-60.

Schneider, B., Cohen, E., Stani, J., Kolbus, A., Rudas, M., Horvat, R. and Trotsenberg, M.V. 2007. Towards the expression of sex hormone receptors in the human vocal fold. J. Voice, 21: 502-507.

Schon, P.C., Hamel, K., Puppe, B., Tuchscherer, A., Kanitz, W. and Manteuffel, G. 2007. Altered vocalization rate during the estrous cycle in dairy cattle. J. Dairy Sci., 90: 202-206.

Srivastava, A.K., Kumarsean, A., Mohanthy, T.K. and Prasad, S. 2013. Status paper on buffalo estrus biology. ICAR-National Dairy Research Institute, Karnal. 1-24.

Taylor, A.M. and Reby, D. 2010. The contribution of source filter theory to mammal vocal communication research. $J$. Zoology, 280: 221-236.

Villanueva, M.A., Mingala, C.N., Tubalinal, G.A.S., Gaban, P.B.V., Nakajima, C. and Suzuki, Y. 2018. Emerging Infectious Diseases in Water Buffalo: An Economic and Public Health Concern. Emerging Infectious Diseases in Water Buffalo-An Economic and Public Health Concern, pp. 1-54.

Voelter, C., Kleinsasser, N., Joa, P., Nowack, I., Martinez, R., Hagen, R. and Voelker, H.U. 2008. Detection of hormone receptors in the human vocal fold. Euro. Arch. Oto-Rhino-L., 265: $1239-1244$.

Warriach, H.M., McGill, D., Bush, R.D. and Wynn, P.C. 2012. Production and reproductive performance of Nili-Ravi buffalo under field conditions of Pakistan. J. Anim. Plant Sci. Eur Arch Otorhinolaryngol Suppl., 3: 121-124.

Yeon, S.C., Jeon, J.H., Houpt, K.A., Chang, H.H., Lee, H.C. and Lee, H.J. 2006. Acoustic features of vocalizations of Korean native cows (Bos taurus coreanea) in two different conditions. Appl. Anim. Behav. Sci., 101: 1-9. 